\title{
DNA copy number gains in malignant pleural mesothelioma
}

\author{
MASASHI FURUKAWA ${ }^{1}$, SHINICHI TOYOOKA ${ }^{1,2}$, TATSURO HAYASHI $^{1,3}$, HIROMASA YAMAMOTO ${ }^{1,3}$, \\ NOBUKAZU FUJIMOTO ${ }^{4}$, JUNICHI SOH ${ }^{1}$, SHINSUKE HASHIDA ${ }^{1,2}$, KAZUHIKO SHIEN $^{1,2}$, \\ HIROAKI ASANO ${ }^{1}$, KEISUKE AOE ${ }^{5}$, KAZUNORI OKABE ${ }^{3}$, HARVEY I. PASS ${ }^{6}$, \\ KAZUNORI TSUKUDA ${ }^{1}$, TAKUMI KISHIMOTO ${ }^{4}$ and SHINICHIRO MIYOSHI ${ }^{1}$
}

\begin{abstract}
Departments of ${ }^{1}$ Thoracic, Breast and Endocrinological Surgery, and ${ }^{2}$ Clinical Genomic Medicine, Okayama University Graduate School of Medicine, Dentistry and Pharmaceutical Sciences, Okayama, Okayama 700-8558; ${ }^{3}$ Department of Thoracic Surgery, National Hospital Organization Yamaguchi-Ube Medical Center, Ube, Yamaguchi 755-0241; ${ }^{4}$ Department of Internal Medicine, Okayama Rosai Hospital, Okayama, Okayama 702-8055;

${ }^{5}$ Department of Medical Oncology, National Hospital Organization Yamaguchi-Ube Medical Center, Ube, Yamaguchi 755-0241, Japan; ${ }^{6}$ Division of Thoracic Surgery, Department of Cardiothoracic Surgery, New York University Langone Medical Center, NY 10016, USA
\end{abstract}

Received October 15, 2014; Accepted July 30, 2015

DOI: $10.3892 / \mathrm{ol} .2015 .3652$

\begin{abstract}
Malignant pleural mesothelioma (MPM) is a highly aggressive tumor with an extremely poor prognosis. The incidence of MPM is increasing as a result of widespread exposure to asbestos. The molecular pathogenesis of MPM remains unclear. The present study analyzed the frequency of various genomic copy number gains (CNGs) in MPM using reverse transcription-quantitative polymerase chain reaction. A total of 83 primary MPMs and 53 primary lung adenocarcinomas were analyzed to compare the CNGs of EGFR, KRAS, MET, FGFR1 and SOX2. In MPM, the CNGs of EGFR, KRAS, MET, FGFR1 and SOX2 were detected in $12(14.5 \%), 8(9.6 \%), 5(6.0 \%), 4(4.8 \%)$ and $1(1.2 \%)$ of the samples, respectively. In lung adenocarcinomas, the CNGs of EGFR, KRAS, MET, FGFR1 and SOX2 were detected in $21(39.6 \%), 12(22.6 \%), 5(9.4 \%), 10(18.9 \%)$ and $0(0.0 \%)$ of the samples, respectively. The CNGs of EGFR, KRAS and $F G F R I$ were significantly less frequent in the MPMs compared with the lung adenocarcinomas $(\mathrm{P}=0.0018$, 0.048 and 0.018 , respectively). Overall, the MPMs exhibited these CNGs less frequently compared with the lung adenocarcinomas $(\mathrm{P}=0.0002)$. The differences in $\mathrm{CNG}$ between the two tumor types suggested that they are genetically different.
\end{abstract}

Correspondence to: Professor Shinichi Toyooka, Department of Clinical Genomic Medicine, Okayama University Graduate School of Medicine, Dentistry and Pharmaceutical Sciences, 2-5-1 Shikata, Kita, Okayama, Okayama 700-8558, Japan

E-mail: toyooka@md.okayama-u.ac.jp

Key words: malignant pleural mesothelioma, lung adenocarcinoma, copy number, reverse transcription-quantitative polymerase chain reaction

\section{Introduction}

Malignant pleural mesothelioma (MPM) is a tumor derived from the mesothelial cells lining the pleural spaces. MPM has highly invasive and aggressive clinical characteristics. Approximately $80 \%$ of MPM patients have a history of occupational asbestos exposure, which is considered to be a risk factor for the development of the disease (1). The molecular pathogenesis of MPM is not well understood. The most common mutations in MPMs are losses in 9p21, 1p36, $14 q 32$ and $22 q 12$, and gains in $5 p, 7 p$ and $8 \mathrm{q} 24$, which have been detected by comparative genomic hybridization analysis $(2,3)$. Homozygous deletion of the 9p21 locus encoding two critical cyclin-dependent kinase inhibitors, $\mathrm{p} 16^{\mathrm{INK} 4 \mathrm{a}}$ and $\mathrm{p} 15^{\mathrm{INK} 4 \mathrm{~b}}$, have been reported in up to $80 \%$ of MPMs, and this mutation may be of diagnostic utility $(4,5)$. The tumor suppressor neurofibromin 2 is encoded by the NF2 gene, located on chromosome 22q12. Mutations in NF2 are found in $\sim 40 \%$ of MPMs, and heterozygous loss of NF2 is identified in 74\% of MPMs $(6,7)$. Mutations are rare in the TP53 and RAS genes, which are frequently present in epithelial solid tumors $(8,9)$. Epigenetic alterations, such as DNA methylation, have been found in MPMs, which have a different profile compared with lung cancer (10-12). MPMs, particularly of the epithelioid subtype, may be hard to differentiate from adenocarcinoma arising in the lung periphery, and epidemiological evidence indicates that asbestos and smoking are shared risk factors for these diseases $(2,13,14)$. Currently, the differential diagnosis of MM is based on a range of morphological analyses, including a combination of histological and immunohistochemical staining, and electron microscopy $(13,15,16)$.

Cytogenetic studies have been performed on MPMs and adenocarcinomas arising in the lung periphery, however, no chromosomal aberrations specific to either of the tumor types have been identified $(2,14)$.

Reverse transcription-quantitative polymerase chain reaction (RT-qPCR) is a method for evaluating DNA copy number 
changes, including losses, gains and amplifications of DNA sequences (17-19). Copy number gains (CNGs) of EGFR and $K R A S$ have been observed in lung cancer, particularly in adenocarcinoma $(18,20)$. Furthermore, CNGs of FGFRl and SOX2 have been observed in lung cancer, particularly in squamous cell carcinoma (21-25). c-Met was recently reported to be activated in MPM by overexpression or mutations in MET (26), and MET amplification is a known cause of resistance to EGFR-tyrosine kinase inhibitor (TKI) treatment in lung cancer (27). RT-qPCR was used in the present study on 83 primary MPM and 53 primary lung adenocarcinomas to compare the CNGs of EGFR, KRAS, MET, FGFR1 and SOX2.

\section{Materials and methods}

Tumor samples. Surgically resected specimens of 53 lung adenocarcinomas and 83 MPMs (57 epithelioid, 8 sarcomatoid, 15 biphasic, 2 desmoplastic and 1 lymphohistiocytic) were obtained. All the lung adenocarcinomas and 11 of the MPM samples were obtained from Okayama University Hospital (Okayama, Japan). Another 18 MPMs were obtained from Yamaguchi-Ube Medical Center (Ube, Japan), 2 were obtained from Okayama Rosai Hospital (Okayama, Japan) and the remaining 52 were obtained from Karmanos Cancer Center (Detroit, MI, USA). All Japanese samples were collected between March 2002 and September 2011, and all samples from the USA were collected $>10$ years ago. Resected tumors were stored at $-80^{\circ} \mathrm{C}$ until DNA extraction. Permission from the Institutional Review Board and informed consent were obtained at each collection site.

DNA extraction. Genomic DNA was obtained from primary tumors by standard phenol:chloroform (1:1) extraction, followed by ethanol precipitation, or using a DNeasy Tissue kit (Qiagen, Inc., Valencia, CA, USA).

RT-qPCR for copy number evaluation. CNGs of EGFR, KRAS, MET, FGFR 1 and SOX2 genes were determined by RT-qPCR assays using Power SYBR ${ }^{\circledR}$ Green PCR Master Mix (Thermo Fisher Scientific, Waltham, MA, USA), as previously described $(18,19)$. Briefly, samples of $1 \mu \mathrm{l}$ were analyzed per assay using with StepOne Plus Real-Time PCR System (Themo Fisher Scientific). PCR conditions were initial denaturation at $95^{\circ} \mathrm{C}$ for $10 \mathrm{~min}$ followed by 40 cycles of amplification at $95^{\circ} \mathrm{C}$ for $15 \mathrm{sec}$ and $60^{\circ} \mathrm{C}$ for $60 \mathrm{sec}$. The samples were analyzed in triplicate using StepOne Plus RT PCR software (version 2.0; Themo Fisher Scientific) and the LINE1 gene was used as a reference gene for all copy number analyses, as this is the most abundant autonomous retrotransposon in the human genome, constituting $17 \%$. Each amplification reaction was checked for the absence of non-specific PCR products by performing a melting curve analysis. The copy number calculation was conducted using the comparative cycle threshold $(\mathrm{Ct})$ method following validation of the PCR reaction efficiency of EGFR, KRAS, MET, FGFR1, SOX2 and LINE1. The PCR primer sequences for EGFR, KRAS, MET and LINE1 primers have previously been described (17-19). The PCR primer sequences for FGFRl and SOX 2 were designed by Primer 3 plus software and by modification of the sequences. The PCR primer sequences were as follows: FGFR1 forward, 5'-AGC CAC CAC ATG GCA TAC
TT-3' and reverse, 5'-GGT GAC AAG GCT CCA CAT CT-3'; and $S O X 2$ forward, 5'-CGT CAC ATG GAT GGT TGT CT-3' and reverse, 5'-GCC GCC GAT GAT TGT TAT TA-3'. The relative copy number of each sample was determined by comparing the ratio of the target gene to LINE1 in each sample with the ratio of these genes in normal human genomic DNA (EMD Biosciences, Darmstadt, Germany) prepared from a mixture of human blood cells from 6-8 donors, as a diploid control. Our previous study defined a copy number of $\geq 4$ as a gene gain in cell lines $(17,18)$. However, considering the contamination by non-malignant cells in primary samples (estimated mean per tumor, 50\% tumor cells and 50\% non-malignant cells), the cut-off value of 3 copy numbers rather than 4 was used for primary tumors in this study (17).

Detection of EGFR mutations. The EGFR mutational status was determined using a PCR-based length polymorphism and restriction fragment length polymorphism assay, as previously described (28). Briefly, the common deletions of exon 19 were distinguished from the wild-type based on PCR product length polymorphisms using $12 \%$ polyacrylamide gel electrophoresis (PAGE) and ethidium bromide staining. For the exon 21 L858R mutation, Sau96I digestion, which specifically digests the mutant type, was performed prior to $12 \%$ PAGE.

Statistical analyses. Differences between the two groups were assessed using the $\chi^{2}$ test or Fisher's exact test as required. All data were analyzed using JMP software version 9.0.0 (SAS Institute Inc., Cary, NC, USA). For all analyses, $\mathrm{P}<0.05$ was considered to indicate a statistically significant difference.

\section{Results}

CNGs in MPMs and lung adenocarcinomas. In the 83 MPM samples, the CNGs of EGFR, KRAS, MET, FGFR1 and SOX2 were detected in 12 (14.5\%), 8 (9.6\%), 5 (6.0\%), 4 (4.8\%), and $1(1.2 \%)$ of the samples, respectively. In the epithelioid subtype of MPM (n=57), the CNGs of EGFR, KRAS, MET, FGFR1 and SOX2 were detected in 7 (12.3\%), $5(8.8 \%)$, $3(5.3 \%), 4(7.0 \%)$ and $0(0.0 \%)$ of the samples, respectively. In the other subtypes of MPMs $(\mathrm{n}=26)$, the CNGs of EGFR, KRAS, MET, FGFR and SOX2 were detected in $5(19.2 \%)$, $3(11.5 \%), 2(7.7 \%), 0(0 \%)$ and $1(3.8 \%)$ of the samples, respectively. In the 53 lung adenocarcinomas, the CNGs of EGFR, KRAS, MET, FGFR1 and SOX2 were detected in 21 (39.6\%), $12(22.6 \%), 5(9.4 \%), 10(18.9 \%)$ and $0(0.0 \%)$ of the samples, respectively (Table I; Fig. 1). Three cases of MPMs were demonstrated to have numerous CNGs of EGFR (269, 62 and 14, respectively). The CNGs of EGFR, KRAS and FGFRl were significantly less frequent in the MPMs compared with the lung adenocarcinomas ( $\mathrm{P}=0.0018,0.048$ and 0.018 , respectively). In the epithelioid subtype of MPMs, the CNGs of EGFR were significantly less frequent than those in the lung adenocarcinomas $(\mathrm{P}=0.0018)$, and in other subtypes of MPMs, the CNGs of FGFRl were significantly less frequent compared with those of the lung adenocarcinomas $(\mathrm{P}=0.026)$. In the MPMs, an absence and presence of CNGs were observed in 64 (77.1\%) and $19(22.9 \%)$ of the 83 cases, respectively. In the epithelioid MPMs, absent/present CNGs were observed in $47(82.5 \%)$ and $10(17.5 \%)$ of the 57 cases, respectively. In the other subtypes of 
Table I. CNGs of EGFR, KRAS, MET, FGFR1 and SOX2 in MPMs and lung adenocarcinomas.

\begin{tabular}{|c|c|c|c|c|c|c|c|c|}
\hline \multirow[b]{3}{*}{ Genes } & \multicolumn{6}{|c|}{ MPMs (n=83) } & & \\
\hline & \multicolumn{2}{|c|}{ All $(n=83)$} & \multicolumn{2}{|c|}{$\begin{array}{c}\text { Epithelioid } \\
\text { subtype }(n=57)\end{array}$} & \multicolumn{2}{|c|}{$\begin{array}{c}\text { Other } \\
\text { subtypes }(n=26)\end{array}$} & \multicolumn{2}{|c|}{$\begin{array}{l}\text { Lung } \\
\text { adenocarcinoma }(\mathrm{n}=53)\end{array}$} \\
\hline & No. & $\%$ & No. & $\%$ & No. & $\%$ & No. & $\%$ \\
\hline$E G F R$ & $12^{\mathrm{a}}$ & 14.5 & $7^{\mathrm{a}}$ & 12.3 & 5 & 19.2 & 21 & 39.6 \\
\hline$K R A S$ & $8^{b}$ & 9.6 & 5 & 8.8 & 3 & 11.5 & 12 & 22.6 \\
\hline$M E T$ & 5 & 6.0 & 3 & 5.3 & 2 & 7.7 & 5 & 9.4 \\
\hline$F G F R 1$ & $4^{\mathrm{b}}$ & 4.8 & 4 & 7.0 & $0^{\mathrm{b}}$ & 0.0 & 10 & 18.9 \\
\hline SOX2 & 1 & 1.2 & 0 & 0.0 & 1 & 3.8 & 0 & 0.0 \\
\hline
\end{tabular}

CNGs of FGFRI and KRAS were significantly less frequent in MPMs compared with lung adenocarcinomas. In epithelioid MPMs, CNGs of $E G F R$ were found to be significantly less frequent compared with lung adenocarcinomas. In other types of MPMs, CNGs of $F G F R 1$ were found to be significantly less frequent compared with lung adenocarcinomas ( ${ }^{\mathrm{a}} \mathrm{P}<0.05$; $\left.{ }^{\mathrm{b}} \mathrm{P}<0.01\right)$. CNGs, copy number gains; MPMs, malignant pleural mesotheliomas.

Table II. Frequency of the absence or presence of CNGs in MPMs and lung adenocarcinomas.

\begin{tabular}{llllr}
\hline & \multicolumn{2}{c}{ Absence of CNGs } & \multicolumn{2}{c}{ Presence of CNGs } \\
\cline { 2 - 3 } Cancer type & No. & $\%$ & No. & $\%$ \\
\hline Malignant pleural mesothelioma $(\mathrm{n}=83)^{\mathrm{a}}$ & $64^{\mathrm{a}}$ & 77.1 & 19 & 22.9 \\
${\text { Epithelioid subtype }(\mathrm{n}=57)^{\mathrm{a}}}_{\text {Other subtypes }(\mathrm{n}=26)}$ & $47^{\mathrm{a}}$ & 82.5 & 10 & 17.5 \\
Lung adenocarcinoma $(\mathrm{n}=53)$ & 17 & 65.4 & 9 & 34.6 \\
\hline
\end{tabular}

Frequency of none of CNGs in MPMs and epithelioid MPMs was significantly higher compared with lung adenocarcinomas ( $\left.{ }^{\mathrm{a}} \mathrm{P}<0.01\right)$. CNGs, copy number gains; MPM, malignant pleural mesothelioma.

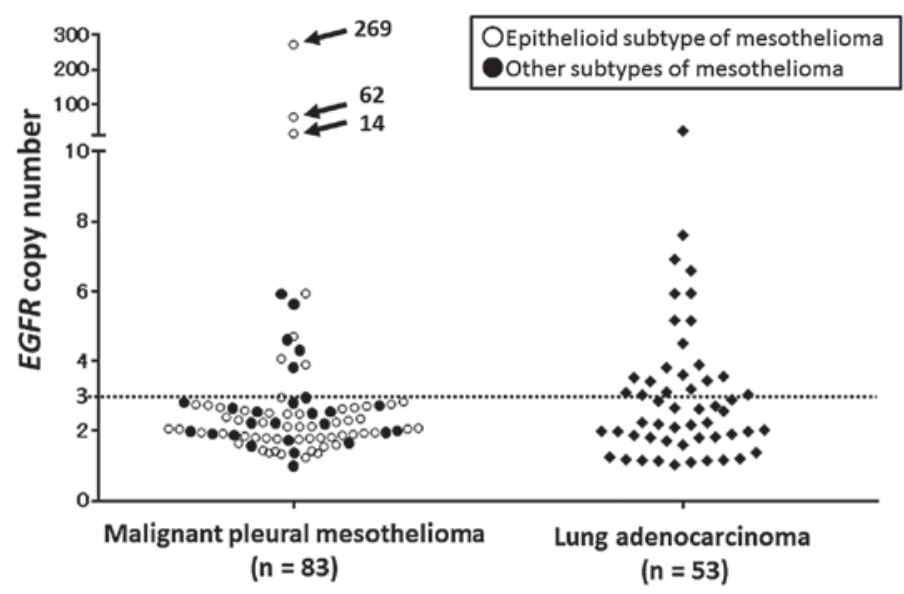

Figure 1. EGFR gene copy number, determined by reverse transcription-quantitative polymerase chain reaction in malignant pleural mesotheliomas (MPMs) and lung adenocarcinomas. Copy numbers $>3$ were considered as copy number gain (CNG). Three cases of MPMs were shown to have high CNGs of $E G F R$ (269, 62 and 14 , respectively).

the MPMs, the absence and presence of CNGs were observed in $17(65.4 \%)$ and $9(34.6 \%)$ of the 26 cases, respectively. In the lung adenocarcinomas, the absence and presence of CNGs were observed in $24(45.3 \%)$ and $29(54.7 \%)$ of the 53 cases, respectively (Table II). The MPMs and the epithelioid subtypes of the MPMs had less frequent CNGs than the lung adenocarcinomas $(\mathrm{P}=0.0002$ and $\mathrm{P}=0.0001$, respectively).

EGFR mutations. No EGFR mutation was detected in the 83 MPMs. In the lung adenocarcinomas, EGFR mutations 
were detected in 21 (39.6\%) cases; 14 cases exhibited an exon 19 deletion and 7 cases exhibited an exon 21 mutation (L858R).

\section{Discussion}

The main finding of the present study is that the pattern of DNA CNGs of MPM is different from that in lung adenocarcinoma. MPMs exhibited less CNGs of the genes examined in compared with the lung adenocarcinomas. The epithelioid subtype of MPM, which is often difficult to distinguish from lung adenocarcinoma, similarly exhibited these CNGs less frequently compared with the lung adenocarcinomas. To the best of our knowledge, only a limited number of studies have previously analyzed the presence and frequency of $E G F R$ CNGs in MPMs (2,29-32), and no studies have focused on CNGs of KRAS, MET, FGFR1 or SOX2 in MPM. A large number of samples $(n=83)$ were screened in the present study, whereas the previous studies were based on smaller sample sizes and may have underestimated the true frequency of such CNGs.

Although CNGs of SOX2 were seldom observed in the MPMs and lung adenocarcinomas, the CNGs of the remaining four genes were detected in the MPM samples to a certain extent. The fact that the CNGs of four genes in the MPMs were less frequent in comparison to the lung adenocarcinomas suggested that CNG may not be a pivotal mechanism for the activation of oncogenes in MPMs, and that different mechanisms may be of greater importance. It has been previously reported that EGFR is overexpressed in 60-70\% of MPM tissue specimens; however, it is not overexpressed in the normal mesothelium (29,33). Furthermore, exposure to asbestos fibers is known to cause EGFR aggregation (34). In the present study, $E G F R$, located at 7p12-p13, was the most frequent gene to exhibit CNGs (12 out of 83 MPMs and 20 out of 53 lung adenocarcinomas). Bjorkqvist et al (2) reported similar results, such as gains of genetic material in $5 p, 6 p$ and $7 p$ between MPMs and lung adenocarcinomas. The study detected a gain in $7 \mathrm{p}$ in 7 out of 34 MPMs and 11 out of 30 lung adenocarcinomas (2). MPMs rarely harbor EGFR mutations (31,35-37). There were no EGFR mutations detected in MPMs in the present study, as expected. Upon analysis, three cases of MPMs exhibited high EGFR gene amplification $(\mathrm{CNG}>10)$, and these cases were all epithelioid MPMs, which was consistent with the previous studies by Okuda et al (29) and Enomoto et al (31). It remains unclear whether high-level amplification of EGFR is more prominent in MPMs compared with lung adenocarcinomas, although the frequency of CNGs for EGFR is lower in MPMs compared with lung adenocarcinomas. In MPMs with EGFR amplification, the inhibition of EGFR pathways should exert an antitumor effect. In lung cancer, the results of two randomized phase III trials that compared a placebo to erlotinib or gefitinib treatment indicated that EGFR copy number detected by fluorescence in situ hybridization was the best predictor of survival (38). Patients with colorectal cancer who responded to anti-EGFR treatment with cetuximab or panitumumab exhibited an increased $E G F R$ copy number (39). Although two phase II studies of single-agent EGFR-TKI therapy to treat MPMs failed to demonstrate their clinical efficacy, in the gefitinib trial, 2 of 43 MPM patients responded to gefitinib $(40,41)$. These data suggest that a small proportion of patients (with
EGFR gene amplification) may be candidates for anti-EGFR treatment (29).

In conclusion, the present study detected novel CNGs in genes other than EGFR. MPM samples exhibited these CNGs less frequently compared with lung adenocarcinomas. The differences in DNA CNG between the two tumor types suggested that they are genetically different.

\section{Acknowledgements}

The authors would like to thank Ms. Fumiko Isobe for providing excellent technical support. The study received Grant-in-Aids for the 13 fields of occupational injuries and illnesses of the Japan Labor Health and Welfare Organization.

\section{References}

1. Spirtas R, Heineman EF, Bernstein L, Beebe GW, Keehn RJ, Stark A, Harlow BL and Benichou J: Malignant mesothelioma: Attributable risk of asbestos exposure. Occup Environ Med 51: 804-811, 1994.

2. Björkqvist AM, Tammilehto L, Nordling S, Nurminen M, Anttila S, Mattson K and Knuutila S: Comparison of DNA copy number changes in malignant mesothelioma, adenocarcinoma and large-cell anaplastic carcinoma of the lung. Br J Cancer 77: 260-269, 1998.

3. Taniguchi T, Karnan S, Fukui T, Yokoyama T, Tagawa H, Yokoi K, Ueda Y, Mitsudomi T, Horio Y, Hida T, et al: Genomic profiling of malignant pleural mesothelioma with array-based comparative genomic hybridization shows frequent non-random chromosomal alteration regions including JUN amplification on 1p32. Cancer Sci 98: 438-446, 2007.

4. Chiosea S, Krasinskas A, Cagle PT, Mitchell KA, Zander DS and Dacic S: Diagnostic importance of 9p21 homozygous deletion in malignant mesotheliomas. Mod Pathol 21: 742-747, 2008.

5. Toyooka S, Kishimoto T and Date H: Advances in the molecular biology of malignant mesothelioma. Acta Med Okayama 62: 1-7, 2008.

6. Fleury-Feith J, Lecomte C, Renier A, Matrat M, Kheuang L, Abramowski V, Levy F, Janin A, Giovannini M and Jaurand MC: Hemizygosity of Nf2 is associated with increased susceptibility to asbestos-induced peritoneal tumours. Oncogene 22: 3799-3805, 2003.

7. Nemoto H, Tate G, Kishimoto K, Saito M, Shirahata A, Umemoto T, Matsubara T, Goto T, Mizukami H, Kigawa G, et al: Heterozygous loss of NF2 is an early molecular alteration in well-differentiated papillary mesothelioma of the peritoneum. Cancer Genet 205: 594-598, 2012.

8. Metcalf RA, Welsh JA, Bennett WP, et al: p53 and Kirsten-ras mutations in human mesothelioma cell lines. Cancer Res 52: 2610-2615, 1992.

9. Papp T, Schipper H, Pemsel H, et al: Mutational analysis of N-ras, p53, p16INK4a, p14ARF and CDK4 genes in primary human malignant mesotheliomas. Int J Oncol 18: 425-433, 2001.

10. Toyooka S, Pass HI, Shivapurkar N, Fukuyama Y, et al: Aberrant methylation and simian virus 40 tag sequences in malignant mesothelioma. Cancer Res 61: 5727-5730, 2001.

11. Kobayashi N, Toyooka S, Yanai H, et al: Frequent p16 inactivation by homozygous deletion or methylation is associated with a poor prognosis in Japanese patients with pleural mesothelioma. Lung Cancer 62: 120-125, 2008.

12. Goto Y, Shinjo K, Kondo Y, et al: Epigenetic profiles distinguish malignant pleural mesothelioma from lung adenocarcinoma. Cancer Res 69: 9073-9082, 2009.

13. Addis B and Roche H: Problems in mesothelioma diagnosis. Histopathology 54: 55-68, 2009.

14. Gee GV, Koestler DC, Christensen BC, et al: Downregulated microRNAs in the differential diagnosis of malignant pleural mesothelioma. Int J Cancer 127: 2859-2869, 2010.

15. Betta PG, Magnani C, Bensi T, Trincheri NF and Orecchia S: Immunohistochemistry and molecular diagnostics of pleural malignant mesothelioma. Arch Pathol Lab Med 136: 253-261, 2012 . 
16. Husain AN, Colby T, Ordonez N, Krausz T, Attanoos R, Beasley MB, Borczuk AC, Butnor K, Cagle PT, Chirieac LR, et al; International Mesothelioma Interest Group: Guidelines for pathologic diagnosis of malignant mesothelioma: 2012 update of the consensus statement from the International Mesothelioma Interest Group. Arch Pathol Lab Med 137: 647-667, 2013.

17. Yamamoto H, Shigematsu H, Nomura M, Lockwood WW, Sato M, Okumura N, Soh J, Suzuki M, Wistuba II, Fong KM, et al: PIK3CA mutations and copy number gains in human lung cancers. Cancer Res 68: 6913-6921, 2008.

18. Soh J, Okumura N, Lockwood WW, Yamamoto H, Shigematsu H, Zhang W, Chari R, Shames DS, Tang X, MacAulay C, et al: Oncogene mutations, copy number gains and mutant allele specific imbalance (MASI) frequently occur together in tumor cells. PLoS One 4: e7464, 2009.

19. Kubo T, Yamamoto H, Lockwood WW, Valencia I, Soh J, Peyton M, Jida M, Otani H, Fujii T, Ouchida M, et al: $M E T$ gene amplification or EGFR mutation activate MET in lung cancers untreated with EGFR tyrosine kinase inhibitors. Int J Cancer 124: 1778-1784, 2009.

20. Sasaki H, Hikosaka Y, Kawano O, Moriyama S, Yano M and Fujii Y: Evaluation of Kras gene mutation and copy number gain in non-small cell lung cancer. J Thorac Oncol 6: 15-20, 2011.

21. Zhao X, Weir BA, LaFramboise T, Lin M, Beroukhim R, Garraway L, Beheshti J, Lee JC, Naoki K, Richards WG, et al: Homozygous deletions and chromosome amplifications in human lung carcinomas revealed by single-nucleotide polymorphism array analysis. Cancer Res 65: 5561-5570, 2005.

22. Yuan P, Kadara H, Behrens C, Tang X, Woods D, Solis LM, Huang J, Spinola M, Dong W, Yin G, et al: Sex-determining region Y-Box $2(S O X 2)$ is a potential cell-lineage gene highly expressed in the pathogenesis of squamous cell carcinomas of the lung. PLoS One 5: e9112, 2010.

23. Kohler LH, Mireskandari M, Knösel T, Altendorf-Hofmann A, Kunze A, Schmidt A, Presselt N, Chen Y and Petersen I: FGFRl expression and gene copy numbers in human lung cancer. Virchows Arch 461: 49-57, 2012.

24. Heist RS, Mino-Kenudson M, Sequist LV, Tammireddy S, Morrissey L, Christiani DC, Engelman JA and Iafrate AJ: FGFR1 amplification in squamous cell carcinoma of the lung. J Thorac Oncol 7: 1775-1780, 2012.

25. Sasaki H, Yokota K, Hikosaka Y, Moriyama S, Yano M and Fujii Y: Increased SOX2 copy number in lung squamous cell carcinomas. Exp Ther Med 3: 44-48, 2012.

26. Jagadeeswaran R, Ma PC, Seiwert TY, Jagadeeswaran S, Zumba O, Nallasura V, Ahmed S, Filiberti R, Paganuzzi M, Puntoni R, et al: Functional analysis of c-met hepatocyte growth factor pathway in malignant pleural mesothelioma. Cancer Res 66: 352-361, 2006.

27. Engelman JA, Zejnullahu K, Mitsudomi T, Song Y, Hyland C, Park JO, Lindeman N, Gale CM, Zhao X, Christensen J, et al: MET amplification leads to gefitinib resistance in lung cancer by activating ERBB3 signaling. Science 316: 1039-1043, 2007.

28. Asano H, Toyooka S, Tokumo M, Ichimura K, Aoe K, Ito S, Tsukuda K, Ouchida M, Aoe M, Katayama H, et al: Detection of $E G F R$ gene mutation in lung cancer by mutant-enriched polymerase chain reaction assay. Clin Cancer Res 12: 43-48, 2006.

29. Okuda K, Sasaki H, Kawano O, Yukiue H, Yokoyama T, Yano M and Fujii Y: Epidermal growth factor receptor gene mutation, amplification and protein expression in malignant pleural mesothelioma. J Cancer Res Clin Oncol 134: 1105-1111, 2008.
30. Rena O, Boldorini LR, Gaudino E and Casadio C: Epidermal growth factor receptor overexpression in malignant pleural mesothelioma: Prognostic correlations. J Surg Oncol 104: 701-705, 2011.

31. Enomoto Y, Kasai T, Takeda M, Takano M, Morita K, Kadota E, Iizuka N, Maruyama H, Haratake J, Kojima Y, et al: A comparison of epidermal growth factor receptor expression in malignant peritoneal and pleural mesothelioma. Pathol Int 62: 226-231, 2012

32. Takeda M, Kasai T, Enomoto Y, Takano M, Morita K, Kadota E, Iizuka N, Maruyama $\mathrm{H}$ and Nonomura A: Genomic gains and losses in malignant mesothelioma demonstrated by FISH analysis of paraffin-embedded tissues. J Clin Pathol 65: 77-82, 2012.

33. Destro A, Ceresoli GL, Falleni M, Zucali PA, Morenghi E, Bianchi P, Pellegrini C, Cordani N, Vaira V, Alloisio M, et al: EGFR overexpression in malignant pleural mesothelioma. An immunohistochemical and molecular study with clinico-pathological correlations. Lung Cancer 51: 207-215, 2006.

34. Pache JC, Janssen YM, Walsh ES, Quinlan TR, Zanella CL, Low RB, Taatjes DJ and Mossman BT: Increased epidermal grow th factor-receptor protein in a human mesothelial cell line in response to long asbestos fibers. Am J Pathol 152: 333-340, 1998.

35. Cortese JF, Gowda AL, Wali A, Eliason JF, Pass HI and Everson RB: Common EGFR mutations conferring sensitivity to gefitinib in lung adenocarcinoma are not prevalent in human malignant mesothelioma. Int J Cancer 118: 521-522, 2006.

36. Velcheti V, Kasai Y, Viswanathan AK, Ritter J and Govindan R: Absence of mutations in the epidermal growth factor receptor $(E G F R)$ kinase domain in patients with mesothelioma. J Thorac Oncol 4: 559, 2009.

37. Mezzapelle R, Miglio U, Rena O, Paganotti A, Allegrini S, Antona J, Molinari F, Frattini M, Monga G, Alabiso O, et al: Mutation analysis of the EGFR gene and downstream signalling pathway in histologic samples of malignant pleural mesothelioma. Br J Cancer 108: 1743-1749, 2013.

38. Tsao MS, Sakurada A, Cutz JC, Zhu CQ, Kamel-Reid S, Squire J, Lorimer I, Zhang T, Liu N, Daneshmand M, et al: Erlotinib in lung cancer - molecular and clinical predictors of outcome. N Engl J Med 353: 133-144, 2005.

39. Moroni M, Veronese S, Benvenuti S, Marrapese G, Sartore-Bianchi A, Di Nicolantonio F, Gambacorta M, Siena S and Bardelli A: Gene copy number for epidermal growth factor receptor $(E G F R)$ and clinical response to anti $E G F R$ treatment in colorectal cancer: a cohort study. Lancet Oncol 6: 279-286, 2005.

40. Govindan R, Kratzke RA, Herndon JE, Niehans GA, Vollmer R, Watson D, Green MR and Kindler HL; Cancer and Leukemia Group B (CALGB 30101): Gefitinib in patients with malignant mesothelioma: A phase II study by the Cancer and Leukemia Group B. Clin Cancer Res 11: 2300-2304, 2005.

41. Garland LL, Rankin C, Gandara DR, Rivkin SE, Scott KM, Nagle RB, Klein-Szanto AJ, Testa JR, Altomare DA and Borden EC: Phase II study of erlotinib in patients with malignant pleural mesothelioma: A Southwest Oncology Group Study. J Clin Oncol 25: 2406-2413, 2007. 